\title{
Impacto de la reducción de lluvias en los pastizales alto andinos de Junín-Perú
}

\author{
Impact of reduced rainfall in the high Andean grasslands of Junín-Peru
}

DOI: $10.46932 / \mathrm{sfjdv} 3 \mathrm{n} 1-088$

Received in: Jan 30st, 2021

Accepted in: Feb 1th, 2022

\section{Paul Franklin Unsihuay Vila}

Ing. Ambiental; M.Sc. en Producción Animal. Consultor independiente en temas ambientales. Urb. Villa Vitarte Mz E Lote 18, Ate, Lima, Perú

E-mail: paulunsihuay1@gmail.com

\section{Lucrecia Aguirre Terrazas}

$\mathrm{Ph}$. D. en Ecología de Praderas Naturales (Utah State University, USA)

Laboratorio de Ecología y Utilización de Pastizales. Universidad Nacional Agraria La Molina

Av. La Molina s/n, Lima, Perú

E-mail: laterra@lamolina.edu.pe

\author{
Franklin Delio Unsihuay Tovar \\ Ing. Meteorólogo; Universidad Nacional Agraria La Molina \\ Av. La Molina s/n, Lima, Perú \\ E-mail: funsihuayt@lamolina.edu.pe
}

\section{RESUMEN}

La presente investigación tiene como objetivo principal estimar el impacto de los cambios en los patrones de precipitación sobre la estructura y funcionalidad del ecosistema de pastizal. El lugar donde se llevó a cabo la investigación fue la comunidad de Consac, distrito de Canchayllo, provincia de Jauja. Se simuló tres niveles de disminución de precipitación, para lo cual se construyeron los "rainout shelters"; interceptores de lluvia que constituyeron los tratamientos a 3 niveles de intercepción de lluvia: 0, 30 y 70\%. Con estos equipos, se evaluó la respuesta de la estructura florística del área y de los grupos funcionales a la reducción de las lluvias durante el periodo de octubre de 2015 a junio del 2016, además de la variación del vigor y la productividad de una especie.

Los resultados encontrados muestran que la reducción de los patrones de precipitación analizados tuvo un impacto significativo en la relación porcentual de las gramíneas, graminoides y herbáceas. Se observó que el porcentaje de herbáceas disminuyó hasta casi un 50\% ante un evento de sequía extrema (70\% de intercepción) al finalizar el estudio respecto a las condiciones iniciales, mientras que las gramíneas aumentaron proporcionalmente a mayor nivel de intercepción y los graminoides mostraron una menor variación. Del mismo modo, observamos que los niveles de temperatura disminuyeron considerablemente en los dos eventos de sequía simulados, donde se observó temperaturas muy bajas de $6.3^{\circ} \mathrm{C}$ que tiene un gran impacto en la actividad metabólica de las plantas. Por otro lado, también se encontró que el vigor de las especies claves se vio fuertemente impactada por la reducción de las precipitaciones, lo cual se manifestó también en la productividad de biomasa aérea, que fue mucho menor en las unidades experimentales que tuvieron mayor intercepción de lluvias que en aquellas que recibieron la cantidad normal de precipitaciones.

Estos resultados muestran que el estrés hídrico en los pastizales se manifestó tanto a nivel de grupos funcionales como a nivel de especies, lo cual sugiere que, ante un evento de sequía moderado a extremo, el ecosistema puede alterar rápidamente su estructura original y que probablemente solo aquellas especies 
que desarrollaron mecanismos de adaptación a las sequías podrán persistir más tiempo como parte de la comunidad vegetal.

Palabras Clave: Disminución de precipitación, rainout shelter, composición florística, temperatura de suelo, humedad de suelo, vigor, productividad.

\begin{abstract}
This research aims to estimate the impact of changes in precipitation patterns on the structure and functionality of grassland ecosystems. The study area was the community of Consac, district of Canchayllo, province of Jauja, Peru. Three levels of precipitation were simulated for which rainout shelters were constructed in order to simulate droughts scenarios at three levels of rain interception: 0, 30 and $70 \%$. Response of floristic structure as functional groups to changes in precipitation patterns were evaluated during two years. Soil moisture and temperature were monitored at $15 \mathrm{~cm}$, vigor and productivity were evaluated on two key ecosystem species.

The results showed that the reduction of rainfall patterns had high impact on the percentage relationship of graminoids, pseudo graminoids and herbaceous plants; where the herbaceous percentage was reduced to almost $50 \%$ in the event of extreme drought (70\% interception) at the end of the study in contrast to the initial conditions. Additionally, the graminoids increased proportionally to a higher level of interception and the pseudo graminoids showed a lower variation. We also observed that the temperature levels decreased considerably in the two simulated drought events, where temperature dropped to $6.3{ }^{\circ} \mathrm{C}$, the decrease in soil temperature observed at the extreme drought event had a great impact on the metabolic activity of the plants. On the other hand, it was also found that the vigor of the key species was strongly impacted by the reduction of precipitation, which was also manifested in above biomass productivity, which was much lower in the experimental units that had greater interception of Rainfall than in those that received the normal rainfalls. These results show that water stress in grasslands affected the functional structure as the species response that in an eventual moderate or extreme drought events, the ecosystem could respond rapidly changing its functional structure, and that probably only those species that have developed drought adaptation mechanisms will be able to persist for a long period at the vegetation community.
\end{abstract}

Keywords: Impacts, land use, forests, Vegetation Index, Normalized Difference, Satellite images.

\title{
1 INTRODUCCIÓN
}

La precipitación es un factor abiótico determinante en la productividad y estructura florística de los ecosistemas terrestres. En la actualidad los patrones de precipitación están cambiando y los modelos climáticos sugieren un aumento de la aridez en muchas zonas del planeta (Vergara et al., 2001). A escala mundial, los cambios en los patrones de precipitación pueden manifestarse en incrementos o reducciones, lo último conduce a estados de sequía, a esto se suma, el incremento de la temperatura y la radiación lo que se traduce en limitaciones ambientales importantes para su sostenibilidad (Yahdjian y Sala, 2008).

Gran parte de las proyecciones que utilizan modelos de simulación ponen de manifiesto la complejidad de los patrones de precipitaciones. Para América del Sur, las proyecciones van desde una reducción de 5-10\% para el 2050, hasta un 20-40\% hacia el año 2080 (Yahdjian y Sala, 2008), mientras 
que para los andes centrales del Perú, las proyecciones indican una reducción de los niveles de precipitación entre 5 a 10\% para el año 2030 (Marengo, 2011) y de $30 \%$ para el 2100 (SENAMHI, 2007).

De acuerdo con la Organización de las Naciones Unidas para la Alimentación y la Agricultura (FAO, 2009), los pastizales constituyen uno de los ecosistemas más grandes del mundo y contribuyen a la subsistencia de más de 800 millones de personas, de las cuales, gran parte encuentran en áreas semiáridas susceptibles en gran medida al déficit hídrico; donde toda disminución de los recursos hídricos afectará de manera importante su capacidad de producción y de sostener herbívoros al pastoreo.

El cambio en los patrones de precipitación en el ecosistema de pastizal puede afectarlo de dos maneras: reduciendo el promedio de disponibilidad de agua e incrementando su variabilidad (Yahdjian y Sala, 2006). Las sequías extremas tienen como consecuencia; a corto plazo, la reducción en la producción primaria y; a mediano y largo plazo, cambios en la composición florística (Yahdjian y Sala, 2011). En el Perú existe muy escasa literatura respecto de los cambios que podrían ocurrir a futuro en los pastizales como consecuencia de la reducción de la precipitación; por tanto, la presente investigación tiene como objetivo estimar el impacto provocado por la reducción de la precipitación sobre la composición florística y productividad del ecosistema de pastizal de la sierra central de Junín. Esta información contribuirá a diseñar estrategias para lidiar con los posibles escenarios futuros en cuanto a la sostenibilidad de los pastizales (Grant et al., 2014).

\section{MATERIALES Y MÉTODOS}

Esta investigación se desarrolló en la Unidad de Consac perteneciente a la Sociedad Agrícola de Interés Social “SAIS Túpac Amaru” ubicado en la provincia de Jauja, región Junín. El área experimental abarca una extensión de terreno de $1200 \mathrm{~m} 2$ (30m x 40m), entre las coordenadas UTM 8677252.58 S y

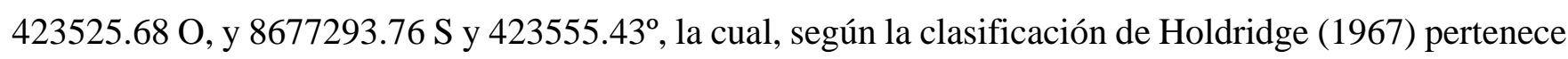
a la zona de vida de Páramo muy húmedo-subalpino tropical. Asimismo, el procedimiento se realizó mediante una lectura diaria a las 7 am desde octubre del 2015 hasta mayo del 2016, tal y como lo muestra la figura 4 (no se registró lluvia durante el mes de junio en ninguno de los dos años de estudio, cuyos resultados indicaron niveles de precipitación registrados de $622.11 \mathrm{~mm}$.

Durante todo el tiempo que duró la investigación, se identificaron un total de 36 especies, de las cuales, al inicio solo se encontraron 13 especies: Calamagrostis Vicunarum, Festuca dolichophylla, Plantago tubulos, Carex sp, Muhlembergia fastigiata, Geranium jaekelae, Lachemilla pinnata, Eryngyum humile, Eryngyum humile, Cerastium crassipes, Azorella compacta y Arenaria digyna. Del mismo modo, se registraron valores de precipitación en el área de estudios mediante un pluviómetro manual. 
El estudio involucró la simulación de tres niveles de suplementación de lluvia, con este fin se construyeron los "rainout shelters" (interceptores de lluvia) con los que se simularon dos escenarios de sequía. Estos interceptores se confeccionaron utilizando piezas de acrílico transparente de 1.85 metros de largo por $11 \mathrm{~cm}$ de ancho con una concavidad en todo el largo para poder drenar el agua de lluvia, donde el área de cada unidad fue de $2.83 \mathrm{~m}^{2}$ (1.85 m x $1.53 \mathrm{~m}$ ); y así, con un techo compuesto de bandas de este material se logró interceptar diferentes cantidades de lluvia mientras que mínimamente afectan a otras variables ambientales.

Este equipo es una adaptación de otro modelo de intercepción de lluvias elaborado por Yahdjian y Sala (2002), quienes los elaboraron para una investigación en los cambios de productividad en la estepa patagónica Argentina (2002). El diseño incluyó cuatro soportes de madera, dos de 1.7 metros y los otros dos de 1.1 metros, con el objetivo de tener una inclinación de $30^{\circ}$ para facilitar la colección de la lluvia. Finalmente, las piezas de acrílico se entornillaron a una estructura de madera y luego esta estructura se entornilló a los soportes mencionados para su armado final. Se colocó una canaleta de metal de $2 \mathrm{~m}$ de longitud en los dos soportes delantales de madera (los de $1.53 \mathrm{~m}$ ) con el fin de acumular el agua interceptada.

Las modificaciones en el diseño se adecuaron de acuerdo a la altura potencial que puede llegar los grupos funcionales de nuestros ecosistemas, especialmente las gramíneas que en su periodo de máximo crecimiento pueden alcanzar 1.3 metros (Tovar, 1993), y se utilizó madera debido a las características climáticas de la zona por la presencia de rayos y la cercanía a cables de alta tensión. Para esta investigación se simularon dos escenarios de sequía, para los cuales se diseñó dos tipos de interceptores de lluvia (figuras 1 y 2) además de un control que recibió la cantidad normal de lluvia, los cuales se describen a continuación:

- Tratamiento 1 (T1): Es el tratamiento control, el que recibirá la cantidad normal de lluvia (100\% precipitación).

- Tratamiento 2 (T2): Diseñado para una reducción de 30\% de las lluvias, por lo cual recibió un nivel de precipitación del $70 \%$ respecto a la cantidad normal.

- Tratamiento 3 (T3): Diseñado para una reducción de 70\% de las lluvias, por lo cual recibió un nivel de precipitación del $30 \%$ respecto a la cantidad normal. 
Figura 1: Modelo de interceptor de lluvia que recibe el 70\% de la precipitación (T2)

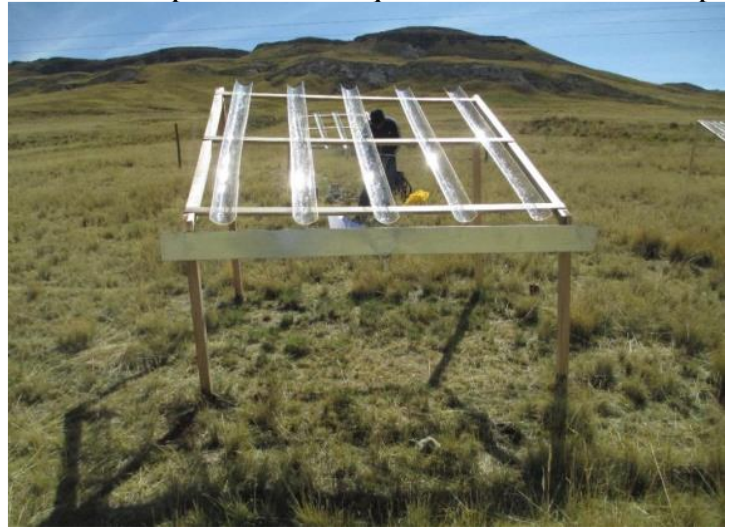

Figura 2: Modelo de interceptor de lluvia que recibe el 30\% de la precipitación (T2)

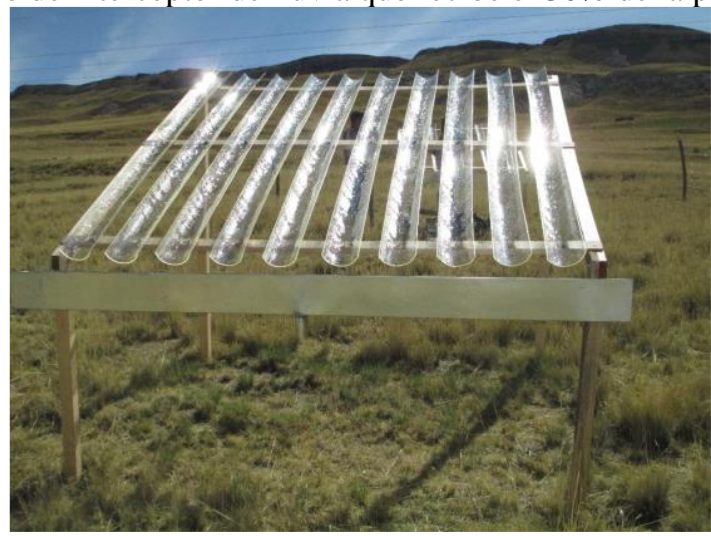

En la figura 1 se muestra el modelo de interceptor para un nivel de precipitación del (70\%), el cual está compuesto de cinco piezas de acrílico, a diferencia de la figura 2 que presenta 10 piezas de acrílico para simular el escenario de sequía más extremo (nivel de precipitación del 30\%). En total, se construyó ocho (8) interceptores de lluvia y las otras cuatro unidades experimentales se marcaron con estacas de madera, las cuales recibieron la cantidad normal del total de lluvias (nivel de precipitación del 100\%). De esta forma, se completaron las 12 unidades experimentales que incluye la investigación.

Por otro lado, la evaluación florística se realizó con el método de la apreciación visual de la cobertura vegetal a nivel de los dos grupos funcionales estudiados: gramíneas y herbáceas. Para estos parámetros, cada unidad experimental se dividió en seis cuadrantes, los cuales fueron elaborados a base de madera y tuvieron dimensiones de $0.45 \mathrm{~m}$ x $0.5 \mathrm{~m}$, tal y como se muestra en la figura 3 . 
Figura 3: Medición de la composición florística

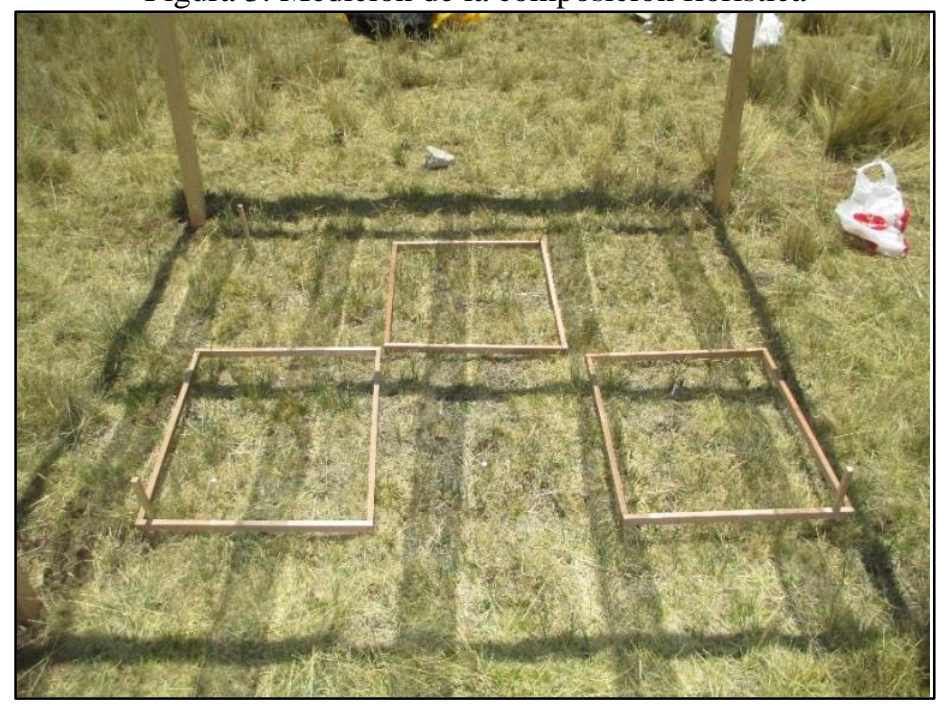

Asimismo, se estimó la variación del volumen de la especie clave elegida en cada una de las épocas de estudio: Festuca dolichophylla. Este volumen, se estimó en función de las medidas de diámetro basal, diámetro de canopia y la altura del esqueje a la hoja bandera, con las cuales se simuló las dimensiones de un tronco de cono invertido bajo la siguiente relación:

$$
\text { Volumen de esqueje planta: } \frac{(D+d)^{2}}{2} \times \pi \times h
$$

Donde:

D: diámetro de canopia

d: diámetro basal

h: altura de hoja bandera

Finalmente, la biomasa de la especie clave fue estimado a partir de los resultados obtenidos del vigor de Festuca dolichophylla, para lo cual se seleccionaron seis ejemplares de la especie clave en tres épocas distintas: inicio de lluvia, pico de lluvia y final de lluvia, cuyas muestras fueron colectadas y llevadas al laboratorio para pesarlas en una balanza electrónica. Con los datos de los pesos medidos y los volúmenes (vigor) calculados con la metodología descrita en el punto anterior, se elaboraron ecuaciones alométricas a partir de un análisis de regresión del peso en función al volumen calculado, la cual se expresa de la siguiente manera:

$$
Y_{1}=\beta_{0} \pm \beta_{1} X_{1} \quad \leftrightarrow \quad \text { peso }=b(\text { volumen }) \pm e
$$

De esta forma, se estimó la producción de biomasa de la especie clave en función a su relación peso-volumen. 


\section{ANALISIS Y DISCUSION DE RESULTADOS}

\section{A) Composición florística}

La composición florística es un parámetro que nos permitió estimar la proporción de especies nativas presente en el área de estudios para luego analizar los cambios a nivel de los dos grupos funcionales identificados para este estudio: Gramíneas y herbáceas. La figura 3 se dividió en 4 partes correspondientes a cada una de las épocas estudiadas donde se realizó la comparación de los dos años de estudio. Como era de esperarse, para cada una de las épocas encontramos que existen diferencias significativas para los tres grupos funcionales $(\mathrm{p}<0.0001)$ y del mismo modo, también encontramos diferencias significativas en los dos años de estudio $(\mathrm{p}<0.0077)$.

Para la época de inicios de lluvia (a), no se observaron muchas variaciones entre los tres grupos funcionales en los tres tratamientos, sin embargo, se muestra que la proporción de herbáceas fue mayor en el T1 (100\% de lluvia recibida) con un $16.81 \%$, mientras que para el T2 (70\% de lluvias) y T3 (30\% de lluvias) se obtuvo 15.84 y $13.32 \%$ respectivamente.

Figura 3: Variación de grupos funcionales a nivel de precipitación recibida y años para cada una de las épocas estudiadas: inicio de lluvias (a), pico de lluvias (b), final de lluvias (c) y época seca (d).

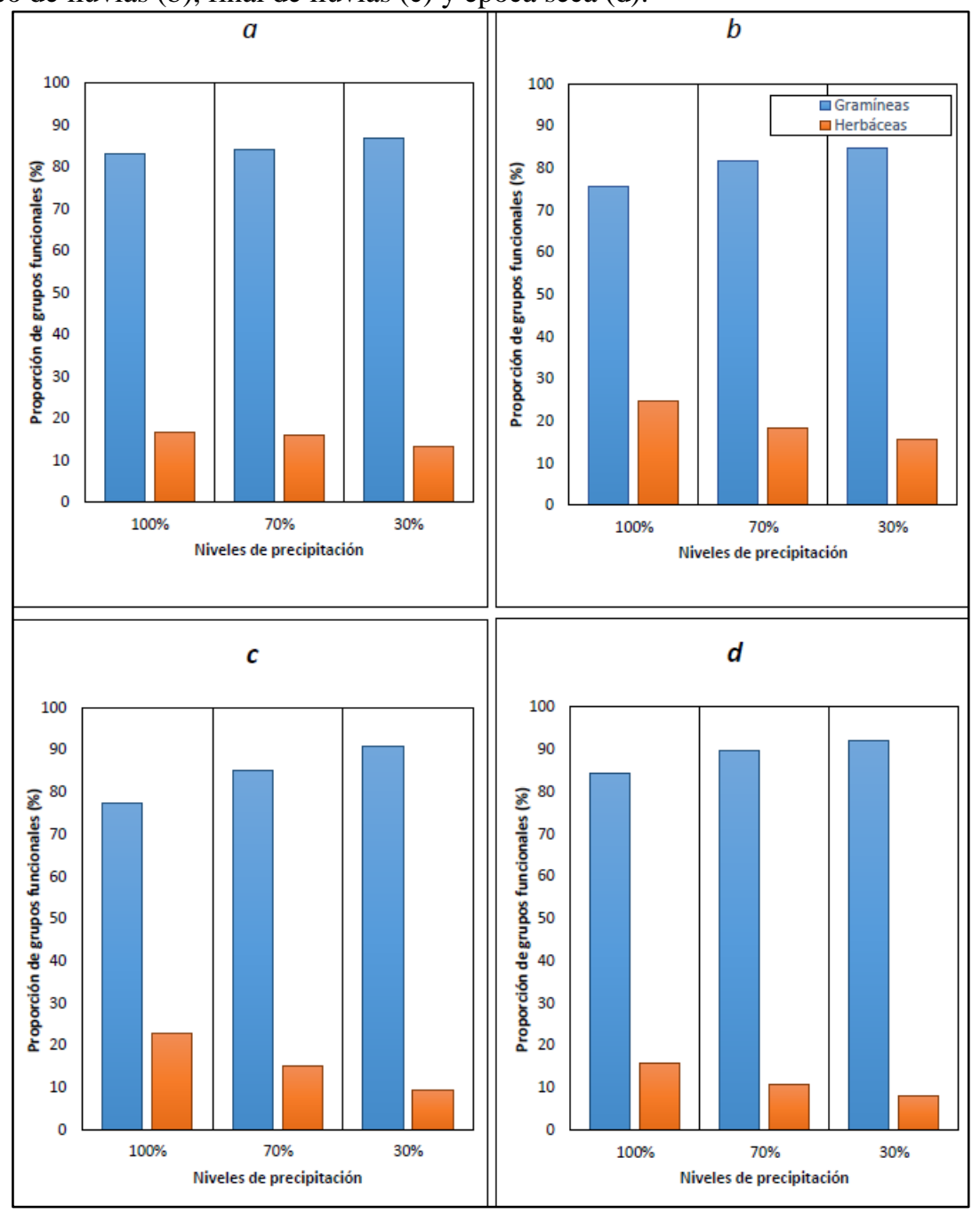


La variación de este grupo en los distintos niveles de precipitación será fundamental para el análisis ya que, al ser especies de porte bajo, tener raíces cortas en su mayoría y además de ser predominada por especies anuales, es el grupo que más se verá afectado por los escenarios de sequía simulados en los tratamientos T2 y T3 (70 y $30 \%$ de lluvia recibida respectivamente). Respecto al grupo de las gramíneas, aparentemente se observa un incremento en los T2 y T3 (81.68 y 84.58\% respectivamente) respecto al T1 (75.47\%), lo cual solo se dio a nivel proporcional mas no cualitativo, pues estos resultados fueron llevados al $100 \%$ para el análisis exclusivo de los grupos funcionales.

Durante la época de pico de lluvia (b), se obtuvieron los mayores porcentajes de herbáceas en el T1 (24.53\%). No obstante, para el T2 y T3, se presentaron mayores reducciones (14.33 y 10.75\%); las cuales indican que, aún en el período de mayor cantidad de precipitaciones de todo el año, los grupos funcionales ven alterados su proporción significativamente tal y como pasó con el período de inicio de lluvias. Para la época de final de lluvia (c) tenemos una disminución significativa de las herbáceas en los tratamientos T2 y T3 (15.04 y 9.2\% respectivamente) respecto al T1 (22.85\%). En ambos casos, la figura indica que el grupo de las gramíneas es mucho mayor en el T2 y T3 (76.05 y 82.88\%, respectivamente) mientras que en el T1 fue de $65.06 \%$.

Para la época seca (d), en el primer año se muestra que las herbáceas disminuyeron drásticamente en todos los tratamientos si los comparamos con los períodos de inicio de lluvia y especialmente en pico de lluvia. Como se observó anteriormente, en el T3 se obtuvo la menor proporción de herbáceas (7.86\%) respecto a los otros dos grupos funcionales y aproximadamente un $40 \%$ menos que su valor inicial $(13.32 \%)$, por tanto, se muestra claramente una tendencia en la variación de los tres grupos funcionales. En cuanto al comportamiento de las gramíneas, si bien es cierto que para los tratamientos T2 y T3 se observa un aumento porcentual el cual alcanza su máximo valor en la época seca que, como lo habíamos mencionado anteriormente, esto no se refleja en un aumento de la cantidad de este grupo, sino refleja la reducción de las herbáceas. Los datos muestran una tendencia clara de la variación y reducción de los grupos funcionales, especialmente de las herbáceas, más aún si se tiene en cuenta que cambios de estos procesos ecológicos se manifiestan con mayor notoriedad en periodos de 3 a 5 años.

Estos resultados reflejan el impacto de las precipitaciones en el crecimiento y desarrollo de las plantas, pues ante eventos de sequía, la disminución de agua afecta al desarrollo y crecimiento de las raíces, las etapas fenológicas e incluso las vías fotosintéticas (Huyghe et al., 2008a, Andueza et al., 2010). También se encontró que un pastizal de estepa patagónica muestra rezagos en su respuesta a una mayor disponibilidad de agua después de un período de sequía (Yahdjian y Sala, 2006). Los rezagos en la recuperación de este pastizal fueron una consecuencia de las restricciones estructurales que la vegetación impuso a la recuperación de la florística de la zona de estudio. 
Durante la sequía, se redujo tanto la producción como la densidad de especies herbáceas y arbustivas. Cuando el agua se hizo disponible después de la sequía, la densidad de las plantas apareció como el principal factor que limitaba la capacidad de la vegetación para responder al aumento de la entrada de agua (Yahdjian y Sala, 2006). Del mismo modo, Golluscio et al. (1998) realizaron un experimento manipulador en la estepa patagónica que encontró que las gramíneas respondían siempre a los grandes eventos experimentales de precipitación de verano, mientras que los arbustos sólo lo hacían al presentarse altas precipitaciones cuando el potencial hídrico del suelo en capas profundas era bajo. La diversidad se redujo en parcelas donde se excluyó el $80 \%$ de la precipitación, lo que se explicó por una reducción en la uniformidad de las especies.

\section{B) Vigor de especie clave}

Como se mencionó anteriormente, las especies claves consideradas para este parámetro fueron Festuca dolichophylla. En la figura 5 se observan los valores de vigor obtenidos para la Festuca dolichophylla, la cual también fue dividida en cada una de sus épocas para un mejor análisis de las variaciones del vigor durante toda la investigación.

Los resultados obtenidos en las figuras 5, mostraron diferencias significativas tanto para los niveles de intercepción $(\mathrm{p}<0.0001)$ y también fue muy significativo para las épocas $(\mathrm{p}<0.0001)$.

En el de inicio de lluvia (a), se nota el efecto de las primeras precipitaciones en el desarrollo de las plantas, pues en el vigor obtenido para esta especie en el T1 (100\% de precipitación) fue de 179816.86 $\mathrm{cm}^{3}$, superior a los que se muestran en el T2 (70\%de precipitación) y T3 (30\% de precipitación) con 14763.44 y $12206.33 \mathrm{~cm}^{3}$. Si bien se observan diferencias entre 2500 a $5000 \mathrm{~cm}^{3}$ entre cada uno de los valores obtenidos anteriormente, estas no se reflejaron al momento de la realización de toma de datos ya que las plantas tuvieron dimensiones similares de diámetro basal, diámetro de canopia y altura de hoja bandera, lo cual se predecía por ser la primera toma de datos. No obstante, los resultados reflejaron el efecto de la reducción de las primeras lluvias en el crecimiento de las plantas. Esto nos muestra que el vigor de las especies no vuelve a recuperarse después de un evento de sequía, incluso cuando la planta recibió el nivel normal de precipitaciones.

Para la época de pico de lluvias (b), se registraron los mayores valores de vigor, específicamente a $100 \%$ de precipitación $\left(63711.5 \mathrm{~cm}^{3}\right)$, mientras que a $70 \%$ de precipitación se obtuvo $40511.56 \mathrm{~cm}^{3}$ y a $30 \%$ se obtuvo $34326.61 \mathrm{~cm}^{3}$. Esta tendencia se repitió incluso con mayor intensidad para el final de lluvias (c), donde los valores obtenidos fueron de $50246.26 \mathrm{~cm}^{3}$ para $100 \%$ de precipitación, 36521.60 $\mathrm{cm}^{3}$ para $70 \%$ de precipitación y $24065.97 \mathrm{~cm}^{3}$ a $30 \%$ de precipitación. 
Figura 5: Variación de los valores de vigor de la Festuca dolichophylla para cada una de las épocas estudiadas: inicio de lluvias (a), pico de lluvias (b), final de lluvias (c) y época seca (d).

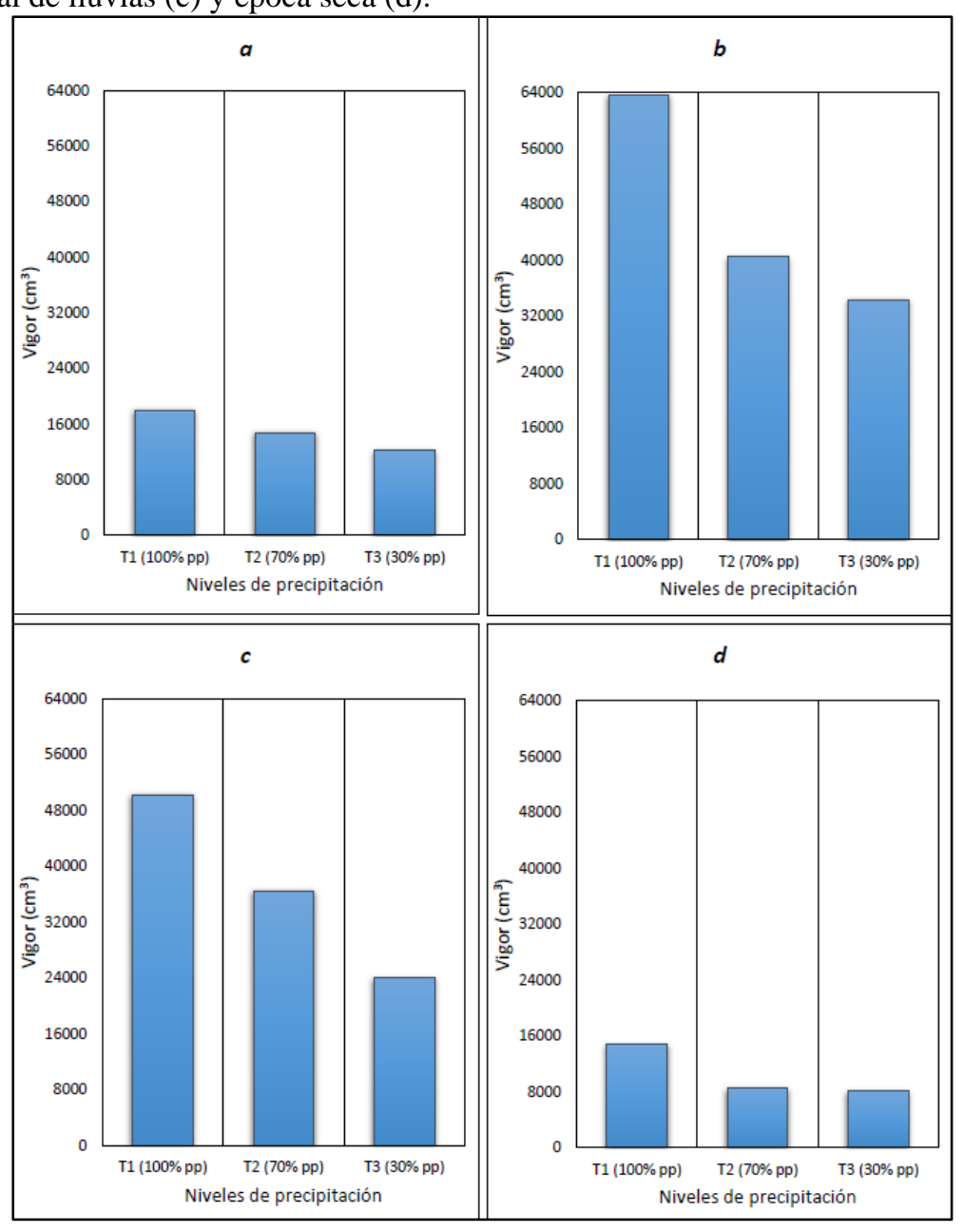

En la época seca (d) también se encontraron reducciones drásticas de vigor en todos los tratamientos, los cuales fueron del orden de 18, 43 y 34\% menos en los tres niveles de precipitación (100, 70 y $30 \%$ respectivamente si los comparamos con los datos de inicios de lluvias, lo cual pudo ser contrastado con la comparación del desarrollo de las plantas en los tres tratamientos.

Estos resultados reflejan un gran impacto de los eventos de sequía simulados en esta especie, y también de las diferencias altamente significativas que se dan en las cuatro épocas; sí se observa una tendencia clara del impacto de la reducción de las precipitaciones en el vigor de la Festuca dolichophylla, que es una especie muy importante tanto a nivel ecológico, como forrajero, pues es una de las más consumidas en la producción ganadera.

\section{C) Biomasa}

Los resultados de biomasa fueron obtenidos mediante ecuaciones alométricas que se desarrollaron descritos en el capítulo anterior, donde se muestra los valores para Festuca dolichophylla. En la figura 6, 
se muestran diferencias significativas para todos los tratamientos $(\mathrm{p}<0.0001)$, donde observamos una tendencia similar en ambas especies. Así mismo, también se encontró diferencias significativas entre las épocas estudiadas $(\mathrm{p}<0.0001)$.

Encontramos valores de biomasa muy parecidos en los tres tratamientos para el primer año en el inicio de lluvias (a), aunque, de todas maneras, la biomasa obtenida a 100\% de precipitación fue el valor más alto (17.49 gr.) respecto a los obtenidos a 70\% de precipitación (16.23 gr.) y a 30\% de precipitación (15.91 gr).

En la época de pico de lluvia (b), se nota una mayor variación de los valores de biomasa en los tres tratamientos, especialmente a 100\% de precipitación (35.81 gr.) respecto a los otros dos de 70 y $30 \%$ (26.53 y 24.05 respectivamente). Esto resulta muy interesante, pues muestra que, aún con la mayor disponibilidad de suplemento hídrico, existe una diferencia más marcada entre el control y las unidades sometidas a intercepción de lluvias, lo cual indica la importancia de las primeras precipitaciones en el crecimiento celular, absorción de nutrientes y metabolismo de los pastizales.

Para el final de lluvias (c) se obtuvieron los descensos más significativos en los tres tratamientos (entre 4 a 5 gramos aproximadamente para cada tratamiento), que fueron de $22 \%$ para el T1 (100\%), 36\% para el T2 (70\% de precipitación) y 27\% para el T3 (30\% de precipitación). En la época seca (d), las variaciones disminuyeron en todos los tratamientos, donde las reducciones más altas en el segundo año fueron de $16 \%$ para el T1 y de $13 \%$ para el T2. Se observa una tendencia clara del impacto de la disminución de las lluvias en la biomasa de la Festuca dolichophylla, pues, si se compara los datos obtenidos en la época seca con los registrados a inicios de lluvia, observamos reducciones de 7, 15 y $23 \%$ para cada uno de los tratamientos evaluados (100, 70 y 30\% de precipitación respectivamente), lo cual confirma que la biomasa también se ve fuertemente afectada ante un evento de sequía moderado a extremo.

En una investigación realizada en la estepa de la Patagonia argentina, Yahdjian encontró mayores valores de producción primaria neta aérea (PPNA) total en parcelas que no habían experimentado sequía que en parcelas con un historial de déficit de precipitación. Este resultado se observó tanto en parcelas de regadío, donde simuló un año húmedo, como en parcelas que recibieron la cantidad de precipitación del sitio durante el tercer año experimental. 
Figura 6: Variación de los valores de biomasa de la Festuca dolichophylla para cada una de las épocas estudiadas: inicio de lluvias (a), pico de lluvias (b), final de lluvias (c) y época seca (d).

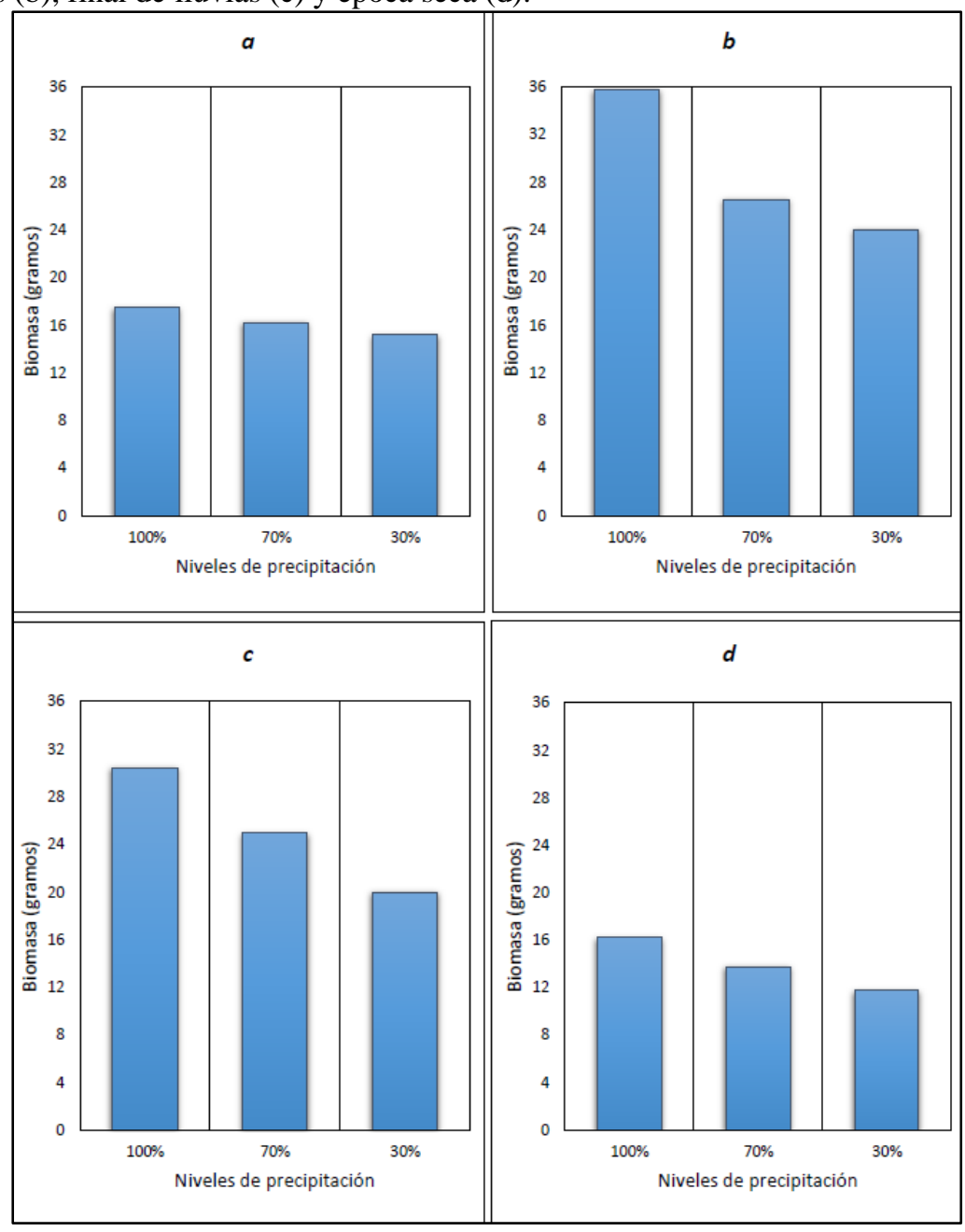

Estos niveles de PPNA también apoyaron la hipótesis de que los retrasos en la recuperación de los PPNA después de los períodos de sequía son proporcionales a la intensidad de la sequía previa. Como consecuencia, el legado relativo de la sequía aumentó con el aumento de la intensidad de la sequía, pues se observó que la PPNA en parcelas que tuvieron $80 \%$ de intercepción (es decir, que solo recibieron un 20\% de lluvia), fue menor que aquellas que tuvieron un 33 y 50\% de intercepción (Yahdjian y Sala, 2006).

\section{CONCLUSIONES}

La proporción de herbáceas disminuyó con la reducción de la precipitación en los dos niveles simulados (70 y $30 \%$ ), en tanto que las gramíneas se aumentaron relativamente en estos dos escenarios. Este efecto varió según las cuatro épocas, cuyas diferencias se hicieron más notorias en la época seca. Asimismo, el vigor de la Festuca dolichophylla se redujo considerablemente en los escenarios de sequía creados (70 y $30 \%$ de precipitación), lo cual se hizo más notorio en el segundo año en la época seca, pues se observó una disminución promedio de 30\% en comparación del inicio de la investigación. Finalmente, 
la biomasa estimada de la Festuca dolichophylla también mostró una tendencia similar a la observada en los resultados de vigor de esta especie. Sin embargo, a diferencia del vigor, las mayores variaciones las encontramos al final de lluvias, aproximadamente un $23 \%$. 


\section{REFERENCIAS}

[1] Aguirre, T. L. 1985. Soil-plant water relation in the altiplano of Peru. M.S. Thesis, Texas Tech University. Lubbock, TX.

[2] Flores A. 1989. Los Pastizales en el Perú. Revista del INIA. 1(3): 15. Chaves MM, Pereira JS, Maroco J, Rodrigues ML, Ricardo CPP, Osório ML, et al. How plants cope with water stress in the field: photosynthesis and growth, Annals of Botany , 2002, vol. 89 (pg. 907-916). Chicahuala S. 2001. "Respuestas fenológicas de dos gramíneas C3 y dos C4, del pastizal natural de San Luis (Argentina), a variaciones de agua y temperatura.", Tesis presentada para optar al título de Magister de la Universidad de Buenos Aires; Recursos naturales-EEA INTA San Luis.

[3] FAO 2009. "Grasslands: enabling their potential to contribute to greenhouse gas mitigation". Informe anual preparado por un grupo de 27 expertos miembros del grupo de trabajo de Pastizales y Carbono en el marco del consejo mundial de la The Food and Agriculture Organization of the United Nations (FAO) Roma, Italia del 15-17 abril 2009.

[4] Golluscio RA, León RJC, Perelman SB (1982) Caracterización fitosociológica de la estepa del Oeste de Chubut; su relación con el gradiente ambiental. Bol Soc Arg Bot 21:299-324.

[5] Heisler-White, Knapp, Kelly Eugene.2008. Increasing precipitation event size increases aboveground net primary productivity in a semi-arid grassland, July.

[6] Holdridge LR (1967) Life zone ecology. Tropical Science Center. San José, Costa Rica. 206 pp.

[7] Instituto Geofísico del Perú, (IGP) 2005. Vulnerabilidad Actual y Futura ante el Cambio Climático y Medidas de Adaptación en la Cuenca del Río Mantaro. Consejo Nacional del Ambiente - CONAM. Av. Guardia Civil 205, San Borja.

Jackson, D.K. (1974). The course and magnitude of water stress in Lolium perenne and Dactylis glomerata. Journal of Agricultural Science (Cambridge), 82: 19-27.

[8] Malpartida E. 1987. Manejo de praderas nativas y pasturas en la región alto andina del Perú. Banco Agrario. Fondo del libro. Tomo I. Lima-Perú. 335 p.

[9] Naupari, V.J. 2000. Variación Estacional en el Consumo, Valor Nutritivo y Perfil Alimentario de Vacas Lecheras en la Unidad de Producción Consac de la SAIS Túpac Amaru Ltda. $\mathrm{N}^{\circ} 1$. Tesis Magister en Producción Animal. Universidad Nacional Agraria La Molina. Lima - Perú.

[10] SENAMHI PERÚ (1988). Mapa de clasificación climática del Perú. Método de Thornthwaite. Eds. Servicio Nacional de Meteorología e Hidrología del Perú, Lima. 9 pp.

[11] Song, Y. T., Zhou, D.W.; Zhang, H.X., Li, G. D., Jin, H. Y., Li, Q., 2013. Effects of vegetation height and density on soil temperature variations. Chinese Science Bulletin. Volume 58, Issue 8, pp 907-912. Article Ecology.

Stampfli, A, Zeiter, M, 2004. Plant regeneration directs changes in grassland composition after extreme drought: a 13-year study in southern Switzerland. J. Ecol.92, 568-576.

[12] Steinaker D.F. y S.D. Wilson. 2008. Phenology of fine roots and leaves in forest and grassland. Journal of Ecology. 96: 1222-1229. 
[13] Tovar.1993. "Las Gramíneas (Poaceae) del Perú” Volumen 13 de Monografías del Real Jardín Botánico Ruizia: Real Jardin Series. CSIC Press.

[14] Vogel, Fester, Eisenhauer, Scherer-Lorenzen, Schmid, Weisser, Weigelt 2013. "Separating Drought Effects from Roof Artifacts on Ecosystem Processes in a Grassland Drought Experiment”.

[15] Yahdjian L., Sala O. E., A rainout shelter design for intercepting different amounts of rainfall, august 2002.

[16] Yahdjian L., Sala O. E. 2008. Climate change impacts on South American rangelands.

[17] Yahdjian L. y Osvaldo 2011. El futuro de los pastizales sudamericanos, feb. Interciencia, Vol. 36 $\mathrm{N}^{\circ} 2$.

[18] Yaranga. 2009. Alimentación de camélidos sudamericanos y manejo de pastizales, Módulo de Camélidos Sudamericanos I, Huancayo, Marzo. 